\title{
Article \\ Endotracheal Tubes Design: The Role of Tube Bending
}

\author{
Talib Dbouk ${ }^{1,2, *(D)}$ and Dimitris Drikakis ${ }^{1}$ (D) \\ 1 Medical School, University of Nicosia, Nicosia CY-2417, Cyprus; drikakis.d@unic.ac.cy \\ 2 Institut Mines-Telecom, 91120 Palaiseau, France \\ * Correspondence: dbouk.t@unic.ac.cy or email@talibdbouk.com
}

check for

updates

Citation: Dbouk, T.; Drikakis, D.

Endotracheal Tubes Design: The Role of Tube Bending. Symmetry 2021, 13, 1503. https://doi.org/10.3390/ sym 13081503

Academic Editors: Iver H. Brevik, John H. Graham and Sergei D. Odintsov

Received: 17 May 2021

Accepted: 4 August 2021

Published: 16 August 2021

Publisher's Note: MDPI stays neutral with regard to jurisdictional claims in published maps and institutional affiliations.

Copyright: (c) 2021 by the authors. Licensee MDPI, Basel, Switzerland. This article is an open access article distributed under the terms and conditions of the Creative Commons Attribution (CC BY) license (https:// creativecommons.org/licenses/by/ $4.0 /)$.

\begin{abstract}
Endotracheal tubes (ETT) passed inside the human trachea witness tube bending at different angles, affecting the local fluid flow dynamics. This induces a variable mechanical ventilation performance across patients' comfortability levels. Our understanding of the local fluid flow dynamics phenomena is thus crucial to enhance the maneuverability of ETT under operation. For the first time to our knowledge, we shed light on ETT through computational fluid dynamics (CFD) to investigate the bending effect of ETT on the local airflow in volume-controlled mechanical ventilation. We considered an ETT with $180^{\circ}$ arc bend configuration, including Murphy's eye. We identified several flow phenomena associated with the bending, such as flow asymmetries, secondary flows, and vortex dynamics throughout the tube.
\end{abstract}

Keywords: endotracheal tube; mechanical ventilation; human health; human respiratory system; modeling and simulation; computational physics; fluid flow dynamics

\section{Introduction}

Endotracheal tubes (ETT) are frequently used in the medical sector to provide mechanical ventilation to patients [1]. They are usually employed for long periods during anesthesia and surgical operations to give oxygen to the human lungs. They were also extensively used in intensive care units of hospitals during the last two years for COVID-19 patients. ETT is manufactured and delivered under different diameters and lengths. Their maneuverability is very complex and constitutes a susceptible procedure for their installation inside the human trachea. This is due to the bending of the tube that must fit well to the mouth-to-trachea internal topology.

ETT aims to cover a wide range of humans trachea tomographies. Farrow et al., 2012 [2] addressed the role of ETT size and its selection depending on the patient's physiology and conditions. Calderon et al., 2019 [3] investigated the mean flow and airway resistance in ETT of several diameters ( 6 to $10 \mathrm{~mm}$ ) at average body temperature and constant pressure numerically. They showed that a single-sized ETT could facilitate endotracheal intubation (ET) without causing an increased airway resistance.

Recent research studies on ETT emerged during the last two years because of the COVID-19 pandemic [4-8]. Piazza et al., 2021 [9] shed light on long-term intubation and the high rate of tracheostomy in COVID-19 patients. Interestingly, they explained how this might determine an unprecedented increase in airway stenoses.

Thanks to advances in computer hardware and CFD methods and models, researchers can investigate the flow phenomena inside the human airways with considerable accuracy. For example, Kou et al., 2018 [10] studied the effect of the cough peak flow rate on airflow dynamics during a cough process in a CT (computerized tomography) scanned human respiratory airway model. Krenkel et al., 2010 [11] developed CFD simulations and experiments to investigate the impact of an ETT on the flow in a generic trachea (artificial lung ventilation). They shed light on models with bendings and connectors that induce more substantial secondary flows that can persist for long periods. Qi et al., 2014 [12] applied CFD simulations to study the airflow mechanisms in the trachea and main bronchi 
for individuals with left pulmonary artery sling. Other scientists used numerical tools to investigate airflow in the human trachea at different conditions $[13,14]$. Mechanical ventilation (MV) has also been a recent topic of research by some investigators [15-20] in attempts to quantify the impact of different MV modes on the aerodynamics of the airways in specific patients.

Kingma et al., 2017 [21] conducted a recent comparison of four methods of endotracheal tube passage in simulated airways. They showed a need for improved techniques to enhance ETT design for better local airflow in the human airways (e.g., human trachea bifurcations) induced by artificial MV.

This study concerns CFD simulations to investigate the airflow dynamics in human trachea first bifurcation from an ETT with Murphy's eye [22-24]. We focus on the ETT vital role in inducing high local airflow circulations inside the trachea with symmetry and asymmetry features of the flow. This work shed light on the importance of appropriate manipulation of ETT during MV that physical practitioners and medical doctors usually manipulate.

\section{Computational Methodology}

We employed the three-dimensional (3D) transient compressible Navier-Stokes equations:

$$
\begin{gathered}
\frac{\partial \rho}{\partial t}+\nabla \cdot(\rho \mathbf{U})=\mathbf{0} \\
\frac{\partial(\rho \mathbf{U})}{\partial t}+\nabla \cdot(\rho \mathbf{U} \otimes \mathbf{U})=-\nabla P+\nabla \cdot \tau \\
\frac{\partial(\rho E)}{\partial t}+\nabla \cdot[(\rho E+P) \mathbf{U}]=\nabla \cdot(\tau \cdot \mathbf{U}),
\end{gathered}
$$

where $\rho, \otimes, \mathbf{U}, P, E, \tau$ represent the fluid density; tensorial product operator; velocity vector; pressure; internal energy and the shear stress tensor, respectively. The system of Equations (1)-(3) is discretized over the computational domain using the Finite Volume Method (FVM) $[25,26]$ inside the open-source CFD code OpenFOAM ${ }^{\circledR}$ [27]. We model turbulence through the Reynolds-Averaged-Navier-Stokes in conjunction with the $k-$ $\omega-$ SST model $[28,29]$. We conducted a mesh sensitivity analysis using three different grids. According to the Grid Convergence Index (GCI) by Celik et al. [30], we adopted a 3D non-uniform hexahedral mesh of about 1 million cells, including local mesh refinement. The trachea geometry is from a commercial provider of a scanned adult patient trachea, transformed into STL surface and treated to retrieve the zone of interest shown in Figure 1c. We applied an inhale-exhale breath with a transient flow rate profile (Figure 1b) imposed at the entry of the ETT extremity. This flow rate mimics a real volume-control (VC) mechanical ventilation signal. A VC ventilation cyclic mode is the most effective one and known to provide full mechanical ventilation. Applying mechanical ventilation with VC mode allows a better control such that for each inspiratory effort beyond a threshold, the ventilator will inject the initially predefined fixed tidal volume. The airflow temperature is imposed $22{ }^{\circ} \mathrm{C}$ at the entry and $33^{\circ} \mathrm{C}$ at the ETT walls. An outlet pressure condition with atmospheric pressure value as a reference and an outlet zero Neumann temperature boundary condition were applied at the two outlets of the trachea (Figure 1). The trachea walls are assumed to be rigid walls with no-slip velocity conditions. 


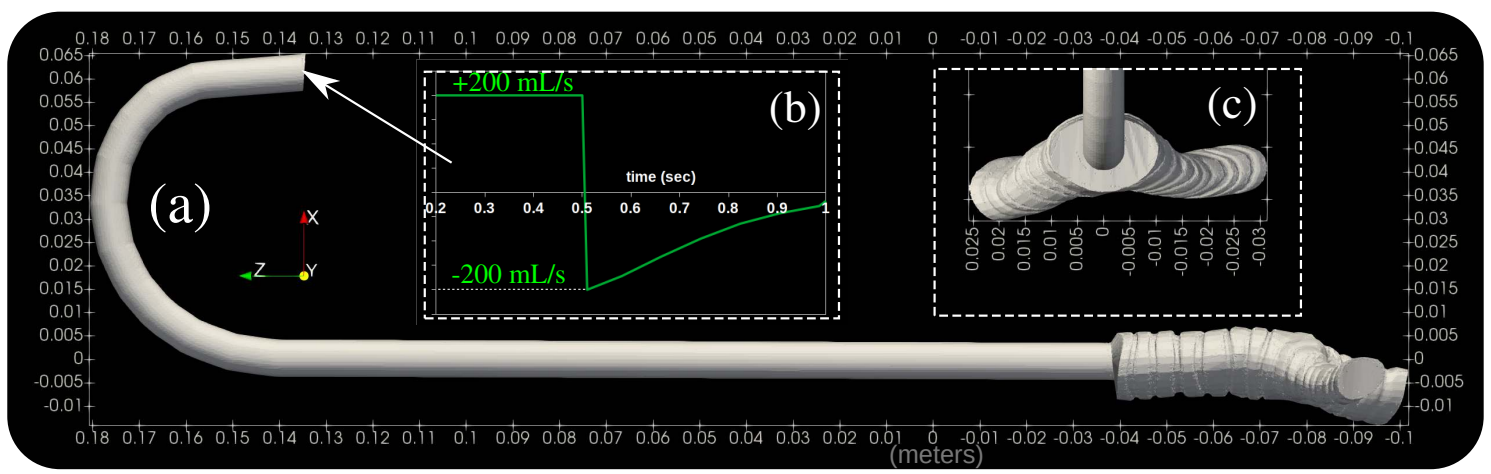

Figure 1. Endotracheal tube (ETT) 3D model of $8 \mathrm{~mm}$ internal diameter inserted into a human's trachea. (a) The computational model used in the simulations; (b) the inhale/exhale applied flow rate boundary condition that mimics a real volume-controlled (VC) mechanical ventilation (MV); (c) upper view of the ETT showing the trachea first bifurcation.

\section{Results and Discussion}

Figure 2 shows the local velocity streamlines in a cross-section post to the ETT extremity and before the bifurcation zone. At the end of the inhale period at $t=0.6 \mathrm{~s}$ (see Figure 2a), airflow asymmetry and small circulations near the trachea wall are observed. We have chosen those two times according to the flow rate cycle shown in Figure 1b. They represent the times at which the highest stresses can occur. The $t=0.5 \mathrm{~s}$ represents the maximum flow speed imposed during the inhalation period just before the exhalation period. The $t=0.6 \mathrm{~s}$ corresponds to the approximate maximum flow speed at the beginning of the exhalation period. At the beginning of the exhale period at $t=0.6 \mathrm{~s}$ (see Figure $2 \mathrm{~b}$ ), airflow asymmetry becomes less manifested with one big circulation (vortex) and the other three smaller vortices near the trachea wall. Note that strong vortices may induce damage to the trachea wall (e.g., inflammation, cracks), especially in patients with MV applied for long periods [9]. Figure 3 illustrates the local streamlines in a cross-section post to the ETT extremity and very close to the first bifurcation zone inside the trachea. At the end of the inhale period at $t=0.5 \mathrm{~s}$ (see Figure 3a), airflow symmetry and four medium to large vortices near the trachea wall are observed. At the beginning of the exhale period at $t=0.6 \mathrm{~s}$ (see Figure $3 \mathrm{~b}$ ), airflow symmetry is still prevailing, but with four small vortices at different positions compared to those observed at $t=0.5 \mathrm{~s}$ in Figure 3a.

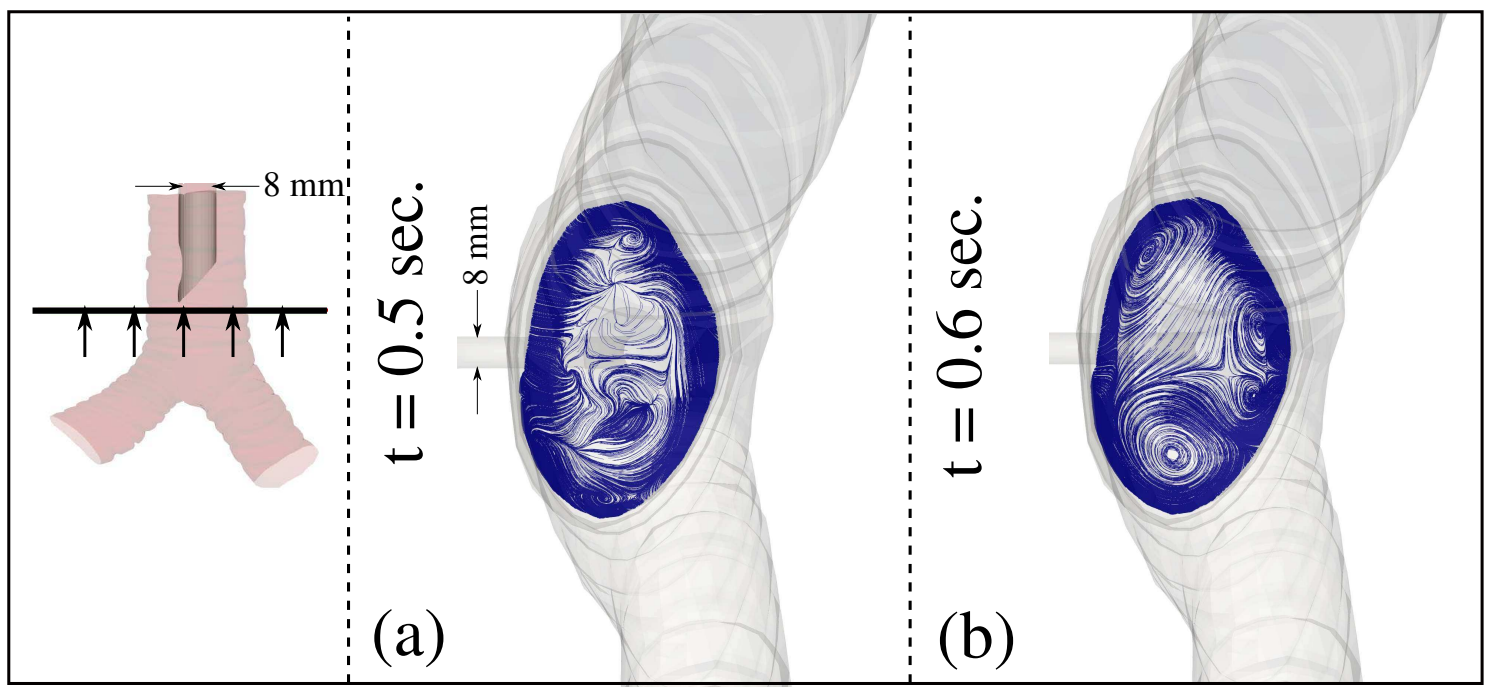

Figure 2. Local velocity streamlines in a cross-section post to the ETT extremity and before the bifurcation zone. (a) Velocity streamlines at the end of the inhale period at $t=0.5 \mathrm{~s} ;(\mathbf{b})$ velocity streamlines at the beginning of the exhale period at $t=0.6 \mathrm{~s}$. 


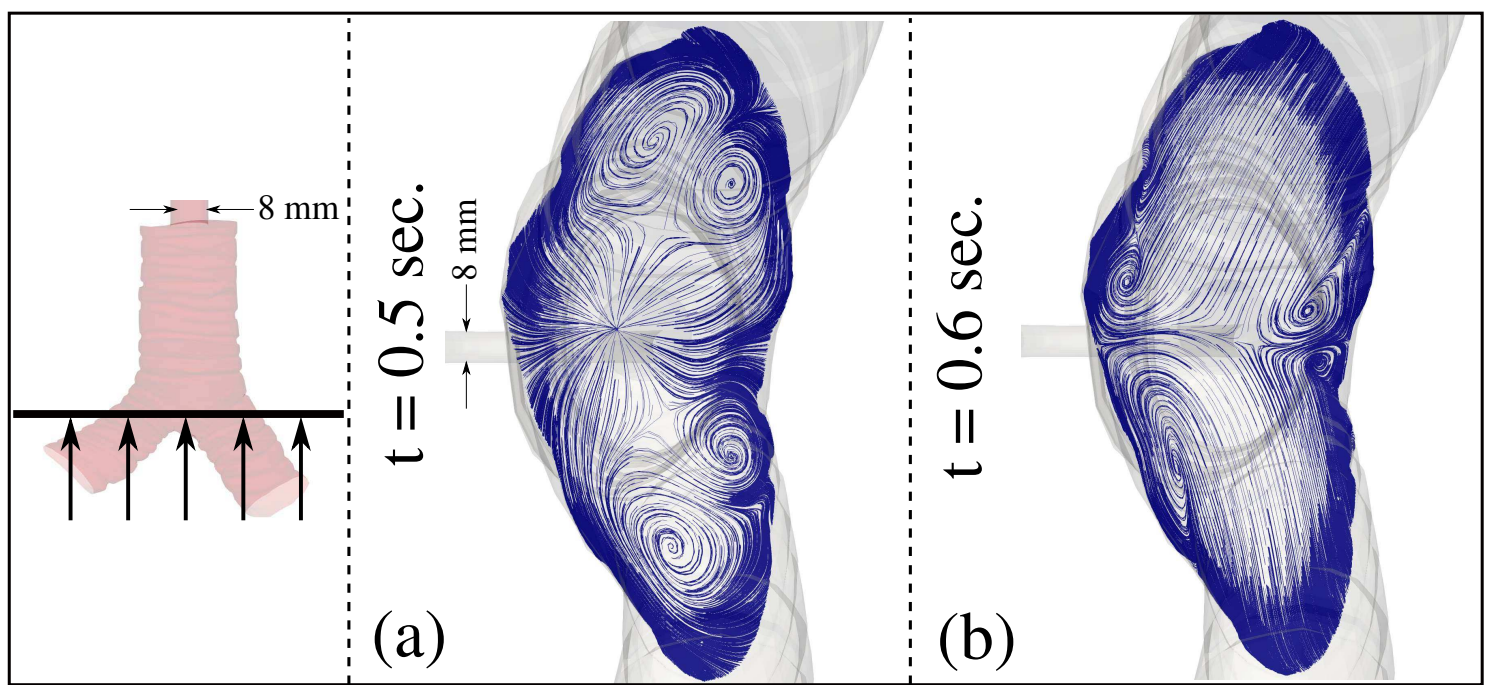

Figure 3. Local velocity streamlines in a cross-section post to the ETT extremity and very close to the bifurcation zone. (a) Velocity streamlines at the end of the inhale period at $t=0.5 \mathrm{~s} ;(\mathbf{b})$ velocity streamlines at the beginning of the exhale period at $t=0.6 \mathrm{~s}$.

Following the bifurcation zone, at the end of inhale period at $t=0.5 \mathrm{~s}$, Figure 4a shows vital airflow circulations with two large vortices in each branch of the trachea. The local $3 \mathrm{D}$ streaklines and WSS are illustrated in Figure 5. Two large vortices at $t=0.5 \mathrm{~s}$ induce significant wall shear stress (WSS), as shown in Figure 5c. These two vortices per branch that were observed at the inhale period at $t=0.5 \mathrm{~s}$ become manifested, but only in the most significant branch addition alone at the beginning of the exhale period at $t=0.6 \mathrm{~s}$ (see Figure $4 b)$.

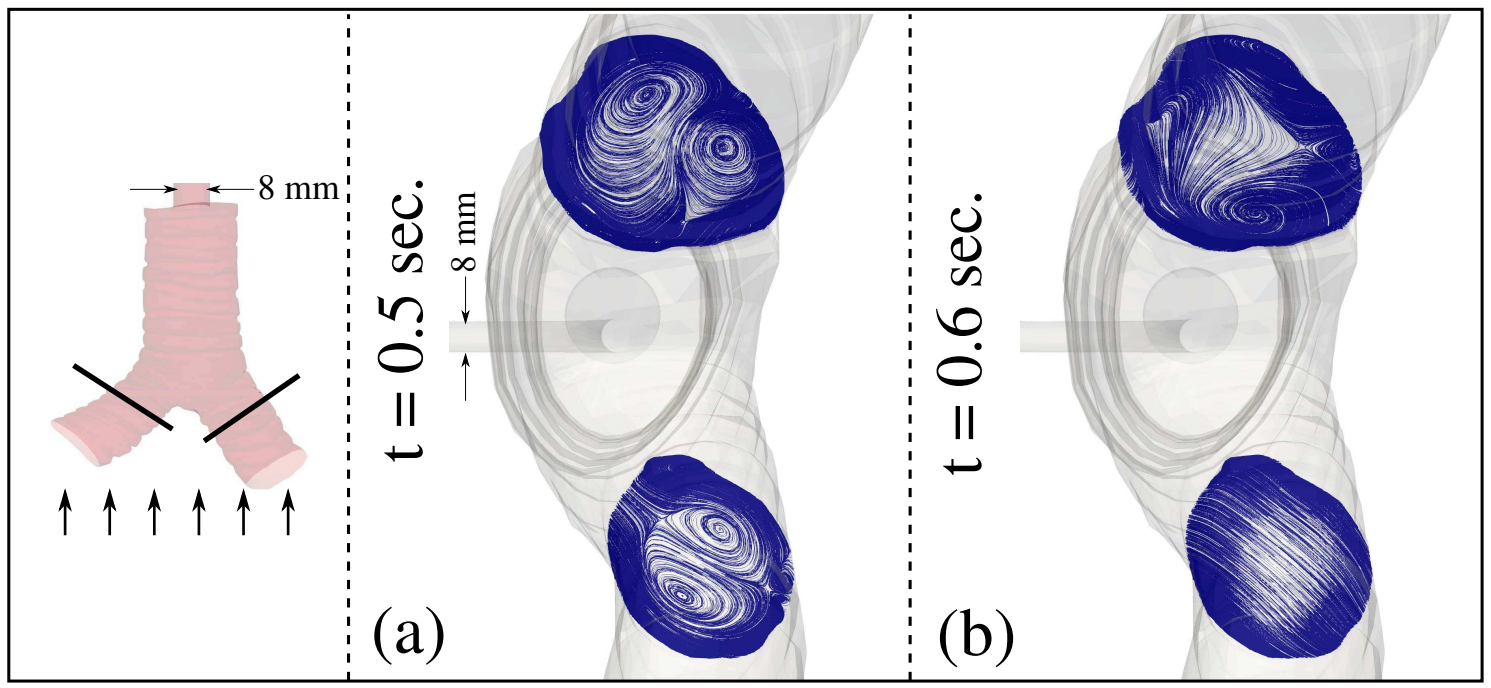

Figure 4. Local velocity streamlines in a cross-section post to the ETT extremity and post to the bifurcation zone. (a) Velocity streamlines at the end of the inhale period at $t=0.5 \mathrm{~s} ;(\mathbf{b})$ velocity streamlines at the beginning of the exhale period at $t=0.6 \mathrm{~s}$.

The WSS values are in the same order of magnitude as those reported in the iterature for an ETT (see Rhein et al., 2016 [15]). They are higher than the WSS values reported in the iterature by Qi et al., 2014 [12] for a subject with a left pulmonary artery sling of the trachea (a numerical study without an ETT [12]). During the inhale phase, the ETT tube of Figure 1 induces more WSS values in the trachea zone following the first bifurcation (Figure 5c,d). In contrast, during the exhale period, it induces more airflow circulations in the trachea zone before the first bifurcation (Figure $5 \mathrm{a}, \mathrm{b}$ ). The flow direction reversibility 
can explain the differences in the streaklines of Figure $5 a, b$, and thus its adaptation to different topologies with the flow direction. In Figure 5a, the flow goes from top to bottom (inhale), thus entering a bifurcation of two outlets, while in Figure 5b, the flow moves from bottom to top (exhale), thus entering Murphy's eye of the ETT.
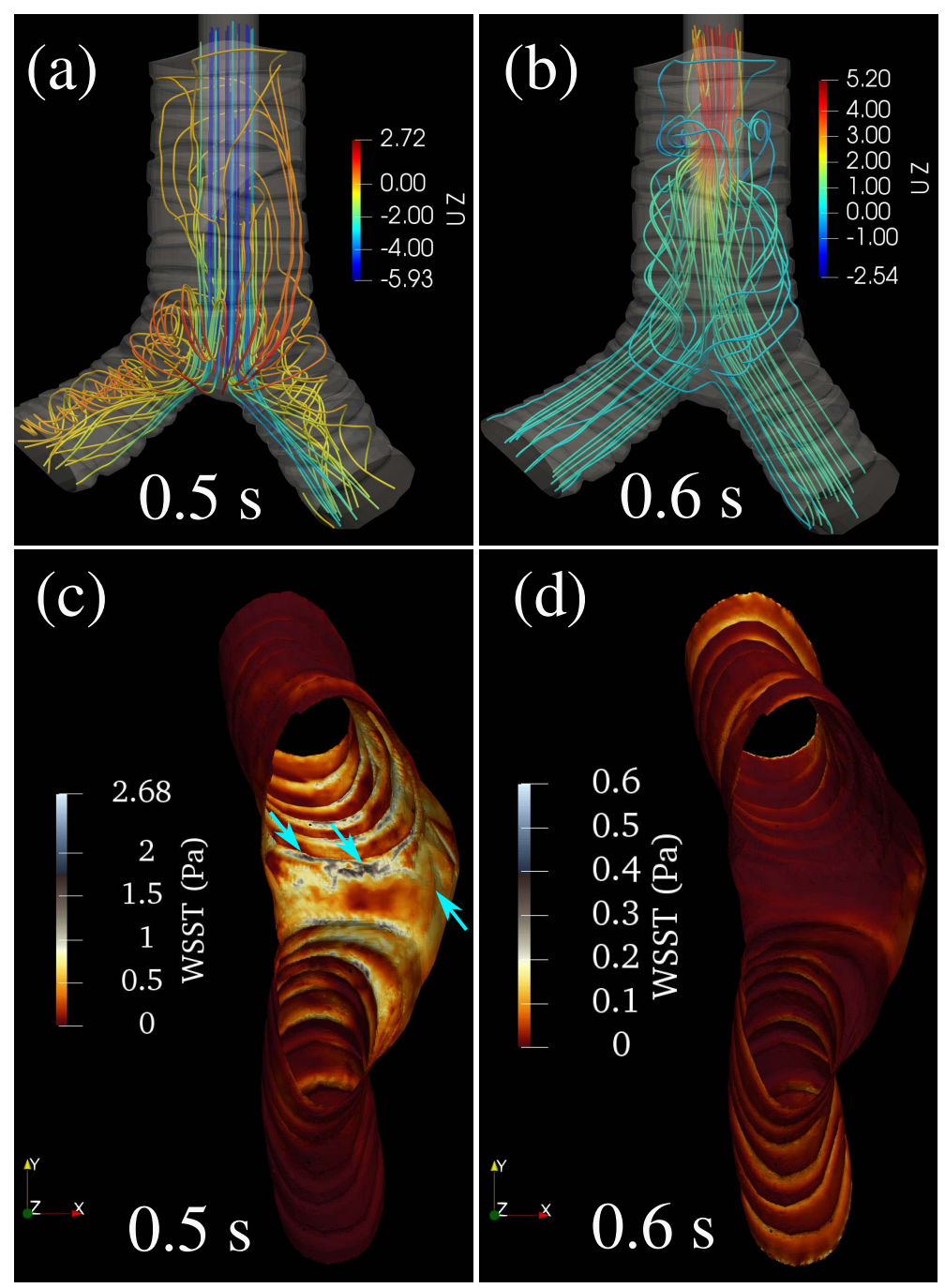

Figure 5. Three dimensional velocity streaklines $(\mathbf{a}, \mathbf{b})$ colored by the axial velocity values $U_{z}(\mathrm{~m} / \mathrm{s})$, and the local wall shear stress (c,d) values WSS (Pa) observed from a top view perspective. (a,c) At the end of the inhale period at $t=0.5 \mathrm{~s} ;(\mathbf{b}, \mathbf{d})$ at the beginning of the exhale period at $t=0.6 \mathrm{~s}$. The small arrows in (c) indicate high local WSS values.

\section{Conclusions and Perspectives}

We shed light on the vital role of ETT bendings in affecting the local airflow in the human trachea during an MV under a VC mode. Employing CFD modeling and simulation, we showed complex local airflow phenomena inside the human trachea that can be observed easily within experiments. In this paper, we focused on an ETT with Murphy's eye and with an arc bending of $180^{\circ}$. Our computations revealed that the ETT tube with this bending angle during the inhale phase induces more WSS values in the trachea zone post to the first bifurcation. Furthermore, our numerical results showed that EET induces more airflow circulations in the trachea zone before the first bifurcation during the exhale period.

The present paper aimed only at raising the question of the bending tube issue through results that demonstrate the complex flow features such as the secondary vortices. At present, the medical practitioner's community are not aware that these effects exist and 
can potentially cause harm to the patient. We aim at studying different configurations and provide a comparative study of different tubes and bendings in a future article.

Author Contributions: Conceptualization, T.D. and D.D.; methodology, T.D.; software, T.D.; formal analysis, T.D. and D.D.; investigation, T.D. and D.D.; resources, T.D. and D.D.; writing-original draft preparation, T.D.; writing—review and editing, D.D.; visualization, T.D.; supervision, D.D. Both authors have read and agreed to the published version of the manuscript.

Funding: This research received no external funding.

Data Availability Statement: All the data supporting reported results are available upon request from the authors.

Acknowledgments: The authors thank the Editorial board for their efforts in publishing this paper.

Conflicts of Interest: The authors declare no conflict of interest.

\begin{tabular}{|c|c|}
\hline \\
\hline \multicolumn{2}{|c|}{$\begin{array}{l}\text { Aboreviations } \\
\text { The following abbre }\end{array}$} \\
\hline CFD & Computational Fluid Dynamics \\
\hline CT & Computerized Tomography \\
\hline ET & Endotracheal Intubation \\
\hline ETT & Endotracheal Tube \\
\hline FVM & Finite Volume Method \\
\hline GCI & Grid Convergence Index \\
\hline MV & Mechanical Ventilation \\
\hline VC & Volume Controlled \\
\hline WSS & Wall Shear Stress \\
\hline
\end{tabular}

\section{References}

1. Benumof, J.L. Airway Management: Principles and Practice; Mosby: St. Louis, MO, USA, 1996.

2. Farrow, S.; Farrow, C.; Soni, N. Size matters: Choosing the right tracheal tube. Anaesthesia 2012, 67, 815-819. [CrossRef]

3. Calderon, L.G.; Moreira, M.M.; Barreto, G.; Tincani, A.J. Model of single-sized endotracheal tube for adults. Einstein 2019, 18, eAO4805. [CrossRef]

4. Gandhi, A.; Sokhi, J.; Lockie, C.; Ward, P.A. Emergency Tracheal Intubation in Patients with COVID-19: Experience from a UK Centre. Anesthesiol. Res. Pract. 2020, 2020, 8816729. [CrossRef]

5. Duggan, L.V.; Mastoras, G.; Bryson, G.L. Tracheal intubation in patients with COVID-19. CMAJ 2020, 192, E607. [CrossRef] [PubMed]

6. Onayemi, A.; Pai, B.H.P. Endo tracheal tube exchange in a COVID positive patient. J. Clin. Anesth. 2020, 66, 109941. [CrossRef]

7. Orser, B.A. Recommendations for Endotracheal Intubation of COVID-19 Patients. Anesth. Analg. 2020, 130, 1109-1110. [CrossRef] [PubMed]

8. World Health Organization. Technical Specifications of Medical Devices for the Case Management of COVID-19 in Healthcare Settings; Technical Report; World Health Organization: Washington, DC, USA, 2020.

9. Piazza, C.; Filauro, M.; Dikkers, F.; Nouraei, S.; Sandu, K.; Sittel, C.; Amin, M.; Campos, G.; Eckel, H.; Peretti, G. Long-term intubation and high rate of tracheostomy in COVID-19 patients might determine an unprecedented increase of airway stenoses: A call to action from the European Laryngological Society. Eur. Arch. Otorhinolaryngol. 2021, 278, 1-7. [CrossRef] [PubMed]

10. Kou, G.; Li, X.; Wang, Y.; Lin, M.; Zeng, Y.; Yang, X.; Yang, Y.; Gan, Z. CFD Simulation of Airflow Dynamics During Cough Based on CT-Scanned Respiratory Airway Geometries. Symmetry 2018, 10, 595. [CrossRef]

11. Krenkel, L.; Wagner, C.; Wolf, U.; Scholz, A.; Terekhov, M.; Rivoire, J.; Schreiber, W. Protective Artificial Lung Ventilation: Impact of an Endotracheal Tube on the Flow in a Generic Trachea. In New Results in Numerical and Experimental Fluid Mechanics VII; Springer: Berlin/Heidelberg, Germany, 2010; pp. 505-512. [CrossRef]

12. Qi, S.; Li, Z.; Yue, Y.; Van Triest, H.; Kang, Y. Computational fluid dynamics simulation of airflow in the trachea and main bronchi for the subjects with left pulmonary artery sling. BioMed. Eng. OnLine 2014, 13, 85. [CrossRef]

13. Wright, P.; Marini, J.; Bernard, G. In vitro versus comparison of endotracheal tube airflow resistance. Am. Rev. Respir. Dis. 1989, 140, 10-16. [CrossRef]

14. Malvè, M.; Chandra, S.; López-Villalobos, J.L.; Finol, E.A.; Ginel, A.; Doblaré, M. CFD analysis of the human airways under impedance-based boundary conditions: Application to healthy, diseased and stented trachea. Comput. Methods Biomech. Biomed. Eng. 2013, 16, 198-216. [CrossRef] [PubMed] 
15. Van Rhein, T.; Alzahrany, M.; Banerjee, A.; Salzman, G. Fluid flow and particle transport in mechanically ventilated airways. Part I. Fluid flow structures. Med. Biol. Eng. Comput. 2016, 54, 1085-1096. [CrossRef] [PubMed]

16. Lumb, A.B.; Burns, A.D.; Figueroa Rosette, J.A.; Gradzik, K.B.; Ingham, D.B.; Pourkashanian, M. Computational fluid dynamic modelling of the effect of ventilation mode and tracheal tube position on air flow in the large airways. Anaesthesia 2015, 70, 577-584. [CrossRef]

17. Lzahrany, M.; Van Rhein, T.; Banerjee, A.; Salzman, G. Fluid flow and particle transport in mechanically ventilated airways. Part II: Particle transport. Med. Biol. Eng. Comput. 2016, 54, 1097-1109. [CrossRef]

18. Zhu, L.; Shen, J.; Gong, X.; Liu, L.; Liu, J.; Xu, Z. Effects of Different Modes of Mechanical Ventilation on Aerodynamics of the Patient-specific Airway: A Numerical Study. In Proceedings of the 2019 41st Annual International Conference of the IEEE Engineering in Medicine and Biology Society (EMBC), Berlin, Germany, 23-27 July 2019; pp. 4961-4964. [CrossRef]

19. Katz, I.; Milet, A.; Chalopin, M.; Farjot, G. Numerical analysis of mechanical ventilation using high concentration medical gas mixtures in newborns. Med. Gas Res. 2019, 9, 213-220. [CrossRef] [PubMed]

20. Yousefi, M.; Safikhani, H.; Jabbari, E.; Yousefi, M.; Tahmsbi, V. Numerical modeling and Optimization of Respirational Emergency Drug Delivery Device using Computational Fluid Dynamics and Response Surface Method. Int. J. Eng. 2021, 34, 547-555. [CrossRef]

21. Kingma, K.; Hofmeyr, R.; Zeng, I.; Coomarasamy, C.; Brainard, A. Comparison of four methods of endotracheal tube passage in simulated airways: There is room for improved techniques. Emerg. Med. Australas. 2017, 29, 650-657. [CrossRef] [PubMed]

22. Forestner, J.E. Frank J. Murphy, M.D., C.M., 1900-1972: His life, career, and the Murphy eye. Anesthesiology 2010, 113, 1019-1025. [CrossRef]

23. Tamakawa, S. Every endotracheal tube needs a Murphy eye! Can. J. Anaesth. 1999, 46, 998-999. [CrossRef]

24. Krzanowski, T.J.; Mazur, W. A complication associated with the Murphy eye of an endotracheal tube. Anesth. Analg. 2005, 100, 1854-1855. [CrossRef]

25. Ferziger, J.H.; Peric, M. Computational Methods for Fluid Dynamics; Springer: Berlin/Heidelberg, Germany, 1999.

26. Moukalled, F.; Mangani, L.; Darwish, M. The Finite Volume Method in Computational Fluid Dynamics: An Advanced Introduction with OpenFOAM and Matlab, 1st ed.; Springer International Publishing: Cham, Switzerland, 2016.

27. Jasak, H. OpenFOAM: Open source CFD in research and industry. Int. J. Nav. Archit. Ocean Eng. 2009, 1, 89-94. [CrossRef]

28. Menter, F. Two-Equation Eddy-Viscosity Turbulence Models for Engineering Applications. AIAA J. 1994, 32, 1598-1605. [CrossRef]

29. Wilcox, D.C. Turbulence Modeling for CFD; DCW Industries, Inc.: La Canada, CA, USA, 2007

30. Celik, I.B.; Ghia, U.; Roache, P.J.; Freitas, C.J. Procedure for Estimation and Reporting of Uncertainty Due to Discretization in CFD Applications. J. Fluids Eng. 2008, 130, 078001. [CrossRef] 